\section{Interventions for intermittent distance exotropia: review}

\begin{abstract}
Purpose Management decisions in intermittent distance exotropia vary and lack well-defined clinical guidelines. We undertook a systematic review in an attempt to clarify the effects of various surgical and nonsurgical treatments and to establish the significance of factors such as age with respect to outcome. The review was undertaken in collaboration with the Cochrane Eyes and Vision Group.

Methods Electronic and manual searches were undertaken to identify randomised controlled trials of surgical or nonsurgical treatments for intermittent distance exotropia. We also contacted researchers active in this field for information about further published or unpublished studies. There were no language restrictions. Study abstracts identified from the searches were analysed independently by the two reviewers (SR and LG) and marked for inclusion, exclusion, or consideration. Reviewer analysis was compared and full papers for appropriate studies were requested.
\end{abstract}

Results No randomised controlled trials were found that met our selection criteria. Conclusions The current literature consists mainly of retrospective reviews. These are difficult to compare and analyse due to variations in definition, intervention criteria, and outcome measures. However, there appears to be an agreement that the nonsurgical treatment is more appropriate in small-angle deviations or as a supplement to surgery. Studies supporting both early and late surgical intervention were found, so the optimal timing of surgical intervention could not be concluded. There is a need for robust clinical trials to improve the evidence base for the management of this condition.

Eye (2005) 19, 617-621. doi:10.1038/sj.eye.6701617

Published online 3 September 2004
L Gnanaraj' and SR Richardson²

Keywords: intermittent exotropia; randomised

control trial; evidence; exotropia

\section{Background}

Strabismus develops in approximately $5 \%$ of developmentally normal children. ${ }^{1}$ Of these, approximately $25 \%$ present with exotropia, ${ }^{2}$ most commonly intermittent distance exotropia (IDEX). The onset of IDEX is usually between 12 months $^{3}$ and 4 years of age and its characteristic features are unique: normal binocular alignment with binocular single vision (BSV) is demonstrable for near fixation, but a constant or intermittent exotropia develops for distance fixation. The total distance angle is usually at least 10 prism dioptres larger than any latent near deviation.

In addition to the trigger of distance fixation, the strabismus may become manifest during periods of inattention, in bright sunlight, or when the subject is tired. Observers may also notice a tendency to close one eye in sunlight. Although subjects with IDEX demonstrate BSV when the eyes are straight, they are rarely symptomatic of diplopia when the deviation is manifest as suppression occurs. ${ }^{4}$

Control for near fixation is thought to be achieved by means of focusing or fusing mechanisms. This has led to IDEX to being subclassified into either 'true' or 'simulated' types: true describes the form where no change in near alignment occurs when proximal fusion and accommodative mechanisms have been released, whereas in the simulated type, the near angle increases to equal or greater than the distance angle either when fusion is disrupted or accommodation is relaxed.

The presenting defect in IDEX is usually the obvious misalignment associated with the exotropia becoming manifest. Visual function, as measured by stereoacuity performance, is
${ }^{1}$ Department of Ophthalmology Royal Victoria Infirmary Newcastle upon Tyne, UK

${ }^{2}$ Department of Orthoptics Royal Victoria Infirmary Newcastle upon Tyne, UK

Correspondence: SR Richardson Department of Orthoptics Royal Victoria Infirmary Queen Victoria Road Newcastle upon Tyne NE1 4LP, UK

Tel.: +441912275206

Fax: +441912820296

E-mail: sarah.richardson@ nuth.northy.nhs.uk

Received: 25 February 2004 Accepted: 29 March 2004

Published online:

3 September 2004

Presented as poster at ARVO May 2003, Royal College Ophthalmologists, June 2003. Published on the Cochrane Library of systematic reviews, April 2003. 
known to be adversely affected during this manifest phase $\mathrm{e}^{5}$ but this is rarely associated with subjective reports of functional deficit. Therefore, the motivation for corrective treatment is most often an improvement in the socially unacceptable nature of the misalignment, rather than any functional gain. This creates a dilemma for the clinician, as any intervention must attempt to reduce the degree of misalignment without detrimentally effecting existing binocular functions.

The aims of intervention in IDEX are to restore orthotropia with BSV for distance viewing while maintaining/improving binocular alignment for near viewing. This can be achieved by reducing the degree of misalignment surgically and/or improving fusional control mechanisms using nonsurgical methods. The indications as to when any intervention should be considered appear to vary, and there are little reliable data on the natural history of the condition; some work has suggested that intermittent exotropia in general is a progressive condition leading to irreversible loss of BSV, while other works suggest that significant numbers improve over time. ${ }^{7}$

Surgery to correct IDEX most commonly involves either unilateral medial rectus resection with lateral rectus recession or bilateral lateral rectus recessions. Unilateral surgery has been thought to be more effective than bilateral surgery in cases where 'simulated' IDEX is present. ${ }^{8}$ The amount of muscle surgery carried out is generally tailored to the size of the deviation, although guidelines for this vary considerably in published surgical tables. Where this is not achieved, subjects are usually either found to be undercorrected with persistence of the intermittent exotropia or, more detrimentally, overcorrected resulting in a loss of BSV with the risk of developing amblyopia. 9,10 This well-documented potential for developing amblyopia as a result of surgical intervention has resulted in some controversy regarding the age at which any surgical intervention should be undertaken: proponents of early intervention ${ }^{11,12}$ (4 years of age or less) argue that despite the increased risk of amblyopia, a functional outcome is more likely to be achieved with early intervention as any sensory defects associated with the strabismus are less entrenched and therefore more easily reversible. Other authorities ${ }^{13,14}$ suggest that sensory abnormalities can and should be controlled by nonsurgical means until an age where surgical intervention can be safely undertaken with reduced risk of any amblyopia development postoperatively. In this way, the age at which surgery is undertaken is considered to have some influence on the outcome of surgery.

Nonsurgical treatment may be undertaken in isolation or as an adjunct to surgery and usually consists of one or a combination of the following:

(1) Orthoptic exercises ${ }^{15}$ to improve the near control of the deviation by increasing fusional reserves. This form of treatment can only be undertaken in older children and is thought to have little effect on the distance deviation.

(2) Part-time occlusion regimes ${ }^{16}$ aim to improve control of the deviation by working against the suppression mechanisms; however, the effect of improved control may be temporary.

(3) Minus lenses ${ }^{17}$ or prisms ${ }^{18}$ can be used to induce convergence, therefore reducing the amount of divergence. However, as most children with IDEX do not otherwise need to wear glasses this treatment may be difficult to persuade patients to accept and any positive treatment effects are again thought to be of limited duration.

Many studies have been published, comparing treatment strategies, ${ }^{9-11,13,17,19,20}$ but despite this there still appears to be a lack of clear evidence to guide clinicians in determining the most effective form of treatment and, if relevant, the age at which any such treatment should be carried out. Our aim in undertaking a systematic review was to report a comprehensive, clear, and unbiased review of the current evidence for the effectiveness of the various treatment modalities in restoring normal ocular alignment and preserving BSV. This was to include reference to any data on the relative effects of age and whether IDEX was of the true or simulated type. The review was undertaken in collaboration with the Cochrane Eyes and Vision Group (CEVG) that, among other things, helped to ensure that the review was conducted thoroughly, systematically, and presented clearly.

\section{Methods}

A protocol was written prior to the commencement of the formal review process in order to reduce the risk of producing a biased and therefore unreliable review. This required prespecification of criteria for the type of study to be included and analysed in the review, what outcome measures would be compared, and how the searches for evidence would be undertaken.

The type of studies stated for inclusion and analysis were randomised-controlled trials (RCTs) comparing management strategies in subjects with IDEX. This level of evidence was chosen, as it is known to provide a more reliable link between intervention and outcome, whereas other study designs are inherently affected by various forms of bias, making the interpretation of the findings less reliable.

As there are other types of intermittent exotropia, any study that did not specify IDEX as a diagnosis and/or 
merged other types of exotropia in the analysis were excluded.

In particular, the review planned to examine the effect of:

(1) Any surgical intervention vs observation alone.

(2) Any nonsurgical vs observation alone.

(3) Surgical vs any nonsurgical.

(4) Unilateral $v s$ bilateral surgery.

(5) Early surgery vs late surgery.

The primary outcome measures were a change in alignment as measured by the degree of squint using the prism cover test or the Synoptophore and demonstration of normal BSV for near and distance as assessed by cover test, fusion range, or stereoacuity.

Adverse effects from any intervention, such as long-term overcorrection of the deviation, were also noted for analysis.

Prestated keywords such as exotropia, intermittent, and divergence were used for all searches. Electronic searches were undertaken by the trial search co-ordinator at CEVG. Trials were searched for in the Cochrane Controlled Trials Register-CENTRAL/CCTR (which contains the CEVG specialised register), the Cochrane Library, MEDLINE, EMBASE, and LILACS (Latin American and Caribbean Literature on Health Sciences). Manual searches on sources known to be inaccessible electronically were also undertaken. This included hand searching the British Orthoptic Journal and proceedings of the following conferences: European Strabismological Association (ESA), International Strabismological Association (ISA), and American Academy of Paediatric Ophthalmology and Strabismus meeting (AAPOS).

Researchers who were known to be active in this field were also contacted for information about further published or unpublished studies. There were no language restrictions in the manual or electronic searches.

The two reviewers independently screened the titles and abstracts obtained by the searches to establish whether they met the inclusion criteria. Where there was agreement between reviewers that a study did not meet the inclusion criteria, it was excluded from further analysis. For abstracts where the reviewers agreed in terms of inclusion, a full copy of the study was obtained. Those where inclusion/exclusion was not clear or the reviewers disagreed in terms of inclusion full copies were also obtained. If the trial was incomplete or unreported, then an attempt was made to contact the investigators to obtain further information.

The study quality was assessed according to the methods set out in the Cochrane Eyes \& Vision Group Methods and Statistics Guidelines. Assessment was made using the following parameters: generation of the random sequence, allocation concealment, masking and completeness of follow-up. Trials were graded as $\mathrm{A}$ - adequate, $\mathrm{B}$ - unclear, or C - inadequate on each parameter.

Full details of the protocol and search methods are available in the published review. ${ }^{21}$

\section{Results}

The electronic searches identified 113 reports of trials on exotropia. In all, 111 of these reports of trials did not fulfil the inclusion criteria, and full text hard copies were obtained for two reports.

In the first of these studies, ${ }^{19}$ patients with basic exotropia were randomised to two different surgical procedures, and these results were compared to patients with simulated IDEX who had all undergone the same procedure (bilateral lateral rectus recession surgery). As this IDEX group was not randomised for their treatment, we were not able to include this study for analysis. The second study ${ }^{20}$ is a prospective RCT, including basic as well as distance types of intermittent exotropia. The results are presented as a whole and not broken down to enable analysis of the IDEX type as a separate entity. This meant that no studies were found that fulfilled the pre-stated inclusion criteria.

\section{Discussion}

The current literature consists mainly of retrospective case reviews and prospective nonrandomised, noncontrolled studies. Although many of these studies present useful data it is difficult to compare and analyse accurately the outcomes due to the wide variation of study designs, diagnostic criteria and outcome measures used.

Data from individual studies on suggested intervention criteria were found to be variable, although there was some consistency in suggesting that smallangle deviations (less than 20 prism dioptres) may be improved by nonsurgical treatments such as exercising fusion, eliminating suppression, or inducing accommodation using minus lenses. However, the efficacy of these treatments remains debatable. ${ }^{22,23}$

Similarly, there was some agreement regarding the criteria that should be considered before undertaking surgery: intervention is recommended if the deviation is noticed at least $50 \%$ of waking hours or if there is deterioration of control for near fixation. ${ }^{24,25}$ There is a controversy in the literature regarding the optimum age for any surgical intervention: Pratt-Johnson et $a l^{11}$ favour early surgery, whereas other authors favour delayed intervention. ${ }^{22,26}$ Pratt-Johnson's prospective study of 100 patients with intermittent exotropia of the divergence 
excess (IDEX) type was designed to address whether the suppression associated with IDEX can be eradicated with surgery and if there was a danger in producing a different type of suppression in overcorrected patients. The definition of sensory 'cure' was strict, stipulating the presence of normal divergence and convergence amplitudes, with diplopia when they were exceeded, and the presence of $40 \mathrm{~s}$ of arc of stereoscopic acuity at near. Of the patients in this study, $81 \%$ were cosmetically satisfactory with a manifest deviation of 10 prism dioptres or less at 1-year postoperation. In all, $41 \%$ were cured, and surgery before the age of 4 years was the most significant factor in obtaining this sensory cure.

However, there was a danger of persistent esotropia in this younger age group.

The type of surgical procedure that should be undertaken has traditionally been agreed as unilateral lateral rectus recession with a medial rectus resection for the simulated type and bilateral lateral rectus recessions for the true type., $, 10,27,28$ However, work by Kushner ${ }^{19}$ found that bilateral lateral rectus recession was effective in correcting the simulated type of distance exotropia. This study evaluated the appropriateness of selective surgery based on distance near differences in the degree of deviation. Subjects with either basic intermittent exotropia or simulated distance exotropia were included. In all, 68 patients with simulated distance exotropia were treated with symmetrical bilateral lateral rectus recessions and of these, $55(80 \%)$ had a satisfactory outcome. Although these subjects were not compared to others undergoing the alternative of unilateral recess/resect surgery, the high success rate from bilateral surgery led to the conclusion that this procedure is effective in correcting simulated distance exotropia.

\section{Conclusion}

As there are no RCTs in this area of clinical practice, it has been difficult to resolve the controversies regarding indications for intervention, the most appropriate treatment option, and the timing of surgery. There is some consistency in the suggested intervention criteria, but there is a clear need for a robust clinical trial to improve the evidence base for the management of this condition.

\section{Acknowledgements}

The CEVG developed and executed the electronic search strategies, guided and monitored the review process, and provided endless support and encouragement.

\section{References}

1 Graham PA. Epidemiology of strabismus. Br J Ophthalmol 1974; 58(3): 224-231.

2 Jenkins R. Demographics geographic variations in the prevalence and management of exotropia. Am Orthopt J 1992; 42: 82-87.

3 Costenbader FD. The physiology and management of divergent strabismus. Strabismic Ophthalmic Symposium, 1950, p 353.

4 Parks M. Sensorial adaptations in strabismus. In: Thomas DD (ed.). Ocular Motility and Strabismus p67. Harper \& Row: Hagerstown, MD, 1975.

5 Stathacopoulos RA, Rosenbaum AL, Zanoni D, Stager DR, McCall L, Ziffer AJ et al. Distance stereoacuity, assessing control in intermittent exotropia. Ophthalmology 1993; 100: 495-500.

6 Rosenbaum A, Stathocopoulos R. Subjective and objective criteria for recommending surgery in intermittent exotropia. Am Orthopt J 1992; 42: 46-51.

7 Hiles D, Davies G, Costenbader F. Long term observations on unoperated intermittent exotropia. Arch Ophthalmol 1968; 80: 436.

8 Burian HM. Selected problems in the diagnosis and treatment of the neuromuscular anomalies of the eyes. In: II Curso internacional de oftalmolgia. Publicaciones del Instituto Barranquer: Barcelona, 1958, p 25.

9 Hardesty HH, Boynton JR, Keenan JP. Treatment of intermittent exotropia. Arch Ophthalmol 1978; 96: 268.

10 Dunlap EA. Overcorrections in horizontal strabismus surgery. In Symposium on Strabismus. Transactions of the New Orleans Academy of Ophthalmology. Mosby Yr Book: St Louis, 1971, p 255.

11 Pratt-Johnson JA, Barlow JM, Tillson G. Early treatment in Intermittent Exotropia. Am J Ophthalmol 1977; 84(5): 689-694.

12 Pratt Johnson JA, Tillson G. Management of Strabismus and Amblyopia: A Practical Guide. Thieme: New York, Stuttgart, 1994.

13 Richard JM, Parks MM. Intermittent exotropia. Surgical results in different age groups. Ophthalmology 1983; 90(10): 1172-1177.

14 Edelman PM, Brown MH, Murphree AL, Wright KW. Consecutive esodeviation Then what? Am Orthopt J 1988; 38: $111-116$

15 Cooper EL, Leyman IA. The management of intermittent exotropia. A comparison of the results of surgical and nonsurgical management. In: Orthoptics: Past Present and Future. Transactions of the $3 r$ International Orthoptic Congress. Stratton Intercontinental Medical Book Corp: New York, 1976.

16 Flynn JT, McKenney S, Rosenhouse M. Eine Behandlungs form des intermittierenden Divergenzschielens [A method of feating intermittent divergence strabismus]. Klin Monatsbl Augenheilk 1975; 167(2): 185-190.

17 Caltrider N, Jampolsky A. Overcorrecting minus lens therapy for treatment of intermittent exotropia. Ophthalmology 1983; 90(10): 1160-1165.

18 Ravault AP, Bongrand G, Bonamour G. The utilization of prisms in the treatment of divergent strabismus. In: Orthoptics. Proceedings of the 2 nd International Orthoptics Congress. Excerpta Medica Foundation: Amsterdam, 1972, p 77.

19 Kushner BJ. Selective surgery for Intermittent Exotropia based on distance near differences. Arch Ophthalmol 1998; 116: 324-328.

20 Kushner BJ. The distance angle to target in surgery for intermittent exotropia. Arch Ophthalmol 1998; 116: 189-194. 
21 Richardson S, Gnanaraj L. Interventions for intermittent distance exotropia (Cochrane Review). In: the Cochrane Library, Issue 2, 2003. Oxford: update software.

22 Rosenbaum AL. Exodeviations. In: Current Concepts in Paediatric Ophthalmology \& Strabismus. University of Michigan: Ann Arbor, MI, 1993, p 41.

23 Von Noorden GK. In: Binocular Vision and Ocular Motility: Theory and Management of Strabismus, 5th edn. Mosby: St Louis, 1996, p 351.

24 Noorden GK von. Some aspects of exotropia. Presented at Wilmer's Residents' Association, John Hopkins Hospital, Baltimore, April 26, 1966.
25 Rosenbaum A, Santiago A. Clinical Strabismus Management. WB Saunders: London, 1999, p 168.

26 Jampolsky A. Management of exodeviations. In: Strabismus. Symposium of the New Orleans Academy of Ophthalmology. Mosby Yr Book: St Louis, 1962, p 43.

27 Burian HM. Exodeviations: their classification, diagnosis and treatment. Am J Ophthalmol 1966; 62: 1161.

28 Burian HM, Spivey BE. The surgical management of exodeviations. Am J Ophthalmol 1965; 59: 603.

29 Burian HM. Exodeviations: their classification, diagnosis and treatment. Am J Ophthalmol 1966; 62(6): 1161-1166. 